\title{
Multiple-Symbol Detection Scheme for IEEE 802.15.4c MPSK Receivers over Slow Rayleigh Fading Channels
}

\author{
Gaoyuan Zhang $\left(\mathbb{D},{ }^{1,2,3}\right.$ Haiqiong Li $\mathbb{D}^{1},{ }^{1}$ Congzheng Han $\mathbb{D}^{2},{ }^{2}$ Congyu Shi $\mathbb{D},{ }^{1}$ Hong Wen, ${ }^{3}$ \\ and Dan Wang ${ }^{1}$ \\ ${ }^{1}$ School of Information Engineering, Henan University of Science and Technology, Luoyang 471023, China \\ ${ }^{2}$ Key Laboratory of Middle Atmosphere and Global Environment Observation, Institute of Atmospheric Physics, \\ Chinese Academy of Sciences, Beijing 100029, China \\ ${ }^{3}$ School of Aeronautics and Astronautics, University of Electronic Science and Technology of China, Chengdu 610054, China
}

Correspondence should be addressed to Gaoyuan Zhang; zhanggaoyuan407@163.com and Congzheng Han; c.han@mail.iap.ac.cn

Received 14 October 2020; Revised 9 November 2020; Accepted 3 May 2021; Published 20 May 2021

Academic Editor: Savio Sciancalepore

Copyright (c) 2021 Gaoyuan Zhang et al. This is an open access article distributed under the Creative Commons Attribution License, which permits unrestricted use, distribution, and reproduction in any medium, provided the original work is properly cited.

\begin{abstract}
Although the full multiple-symbol detection (MSD) for IEEE 802.15.4c multiple phase shift keying (MPSK) receivers gives much better performance than the symbol-by-symbol detection (SBSD), its implementation complexity is extremely heavy. We propose a simple MSD scheme based on two implementation-friendly but powerful strategies. First, we find the best and second-best decisions in each symbol position with the standard SBSD procedure, and the global best decision is frozen. Second, for the remaining symbol positions, only the best and second-best symbol decisions, not all the candidates, are jointly searched by the standard MSD procedure. The simulation results indicate that the packet error rate (PER) performance of the simplified MSD scheme is almost the same as that of the full scheme. In particular, at PER of $1 \times 10^{-3}$, no more than $0.2 \mathrm{~dB}$ performance gap is observed if we just increase the observation window length $N$ to 2 . However, the number of decision metrics needed to be calculated is reduced from 256 to 2 . Thus, much balance gain between implementation complexity and detection performance is achieved.
\end{abstract}

\section{Introduction}

With the widespread application of new information and communication technologies such as the Internet of Things (IoT), cloud computing, and big data, smart cities have developed rapidly in recent years. They have penetrated into all aspects of people's lives and greatly meet the modern people's pursuit of convenient, fast, and high-quality life [1-7]. Reliable and effective transmission of the sensing data is obviously important for the construction of the new smart city [8]. The IEEE $802.15 .4 \mathrm{c}$ protocol provides the physical layer specification of the low-power short-distance IoT for China $[9,10]$. The multiple phase shift keying (MPSK) is provided in IEEE 802.15.4c. This mainly follows from the fact that MPSK modulation is the most able to provide high reliability as well as data rate for sensing data transmission.
Therefore, it is important to study robust detection technology of MPSK signal in line with the characteristics of wireless IoT. This paper focuses on the multiple-symbol detection (MSD) of IEEE 802.15.4c MPSK receiver.

Although the MSD scheme has excellent detection performance, its implementation complexity increases exponentially with the increase of the observation window length $[11,12]$. In recent years, many concentrations have been achieved on complexity reduction of MSD. Stephen et al. studied the maximum likelihood detection (MLD) based on information symbol blocks. The corresponding block signal is used to limit only a part of the possible signal decisions, which will reduce the complexity of the receiver. However, there is a partial performance loss [13]. LoRici proposed a suboptimal receiver based on Viterbi algorithm. The complexity of the receiver increases in polynomial form 
of $M$. However, the performance of the algorithm is related to the memory length $L$ of continuous phase modulation signal. For continuous-phase frequency-shift keying (CPFSK) signal $(L=1)$, its detection performance is seriously degraded $[14,15]$. Several low-complexity MSD algorithms are also proposed by Fischer and Wang Xin, but their performance is far behind that of the traditional MSD algorithm [16, 17].

In this work, we propose a simple MSD scheme for IEEE 802.15.4c MPSK receivers. Unlike the traditional receivers that were equipped with full MSD scheme with high complexity to achieve the best possible reliability, we pay our full attention to the simple design to balance the complexity and reliability. We summarize our main contributions as follows:

(i) The optimal MSD scheme for IEEE 802.15.4c MPSK receivers based on the maximum likelihood criterion can give excellent results in the case of both slow fading and pure additive white Gaussian noise (AWGN) channels. However, the implementation is relatively complex and unachievable for IEEE 802.15.4c MPSK receivers. As an implementation achievable benchmark, a full MSD scheme based on compensation is proposed.

(ii) As for the proposed full MSD scheme, more than twohundred-decision statistic should be calculated before making final decision even if we set the observation window length $N$ to 2 . Thus, we propose a new MSD algorithm, which greatly simplifies the full scheme.

(iii) In order to verify the desirable properties we obtained from this simple scheme, the characteristics of the receiver are studied from many aspects with extensive simulations.

The rest of this paper is organized as follows: Section 2 focuses on the signal model under the slow fading Rayleigh channel. Section 3 describes the full MSD scheme, and Section 4 introduces the proposed simplified MSD scheme. Section 5 concentrates on frequency offset estimation. The simulation results are discussed in Section 6. Finally, some conclusions and future work are provided in Section 7.

\section{System Model}

According to the IEEE $802.15 .4 \mathrm{c}$ protocol [18], the specific data modulation process for the MPSK physical layer is shown in Figure 1. From the binary data of the physical layer protocol data unit (PPDU), in each symbol period, four information bits form a symbol, which is used to select one of 16 orthogonal spreading sequences. The chips in the sequence are MPSK-modulated onto the carrier. For more details on the mapping rules, please refer to Table 1 in [19].

Ideal carrier synchronization is assumed at the receiver. Specifically, for the $x$ th symbol $E[x]$, the received complex baseband chip sequence can be expressed as

$$
r_{x, m}=h_{x, m} s_{y, m} e^{j\left(\omega_{x, m} m T_{c}+\theta_{x, m}\right)}+\eta_{x, m}, \quad 1 \leq m \leq M,
$$

where $h_{x, m}$ represents multiplicative fading, $s_{y, m}$ is the $m$ th chip of the $y$ th pseudorandom (PN) sequence $\mathbf{s}_{y}$, and Table 1 shows the detailed correspondence. $\omega_{x, m}=2 \pi f_{x, m}$ represents the carrier frequency offset (CFO) in radians, and $f_{x, m}$ represents the residual CFO in Hz. $\theta_{x, m}$ represents the carrier phase offset (CPO) in radians, and $T_{c}$ represents the spreading chip period. $\eta_{x, m}$ is a discrete, cyclic symmetric, complex Gaussian random variable with zero mean and variance $\sigma_{x, m}^{2}$, and $M=16$ represents the length of the PN sequence [18].

We assume that a piecewise constant approximation is made to the multiplicative fading, CFO, and CPO [20]. That is, $h_{x, m}=h, \omega_{x, m}=\omega$, and $\theta_{x, m}=\theta$. In addition, the receiver does not have any prior information about the CPO; that is to say, the uniform distribution in the interval $(-\pi, \pi)$ is assigned to $\theta$. The normalized complex Gaussian process $h$ follows Rayleigh distribution; that is, the mean $\bar{h}=0$. The CFO $f$ follows a symmetrical triangular distribution.

\section{The Full MSD Scheme}

Following the idea in [21], we can easily develop the optimal MSD scheme for IEEE 802.15.4c MPSK receivers based on MLD. However, the implementation complexity is extremely heavy as shown in [22], which limits its application in smart cities. Here, we consider a heuristic configuration. The specific detection process is as follows.

First, the baseband chip sample after carrier frequency offset effect (CFOE) compensation can be expressed as

$$
r_{x, m}^{\prime}=r_{x, m} e^{-j m \hat{\varphi}}
$$

where $\widehat{\varphi}=\widehat{\omega T c}$ denotes the estimated CFOE. The estimation of CFOE should be carefully developed and will be described in detail in Section 5. Please note that we assume that the effect of redundant parameter $\omega T_{c}$ on $r_{x, m}$ is completely eliminated after compensation. In addition, the information is embedded in the carrier phase but not in the carrier amplitude. Therefore, there is no need to estimate and compensate for the multiplicative fading $h$ even if serious fading of the received signal strength may be exhibited.

Secondly, we divide the whole compensated chip sequence into block, and each block contains $N$ symbols. The detection metric for the ith block can be then expressed as [23]

$$
U_{\mathbf{x}}=\left|\sum_{x=(i-1) N+1}^{i N} \sum_{m=1}^{16} r_{x, m}^{\prime} s_{y, m}^{*}\right|^{2}, i \geq 1,1 \leq y \leq 16,
$$

where

$$
\begin{aligned}
U_{x}= & \left\{U_{(i-1) N+1,1}, U_{(i-1) N+1,2}, \ldots, U_{(i-1) N+1,16}, U_{(i-1) N+2,1},\right. \\
& \left.U_{(i-1) N+2,2}, \ldots, U_{(i-1) N+2,16}, \ldots, U_{i N, 1}, U_{i N, 2}, \ldots, U_{i N, 16}\right\}
\end{aligned}
$$

and * represents complex conjugate operation. Note that, for $N=1$, (3) reduces to the symbol-by-symbol detection (SBSD) scheme. From (3), we can also see that the multiplicative fading $h$ has no effect on the final decision, and there is no need to estimate and compensate for the fading coefficient $h$.

Finally, the decision rule can be expressed as follows:

$$
\widehat{U}[\mathbf{x}]=\arg \max \left\{U_{\mathbf{x}}\right\} .
$$




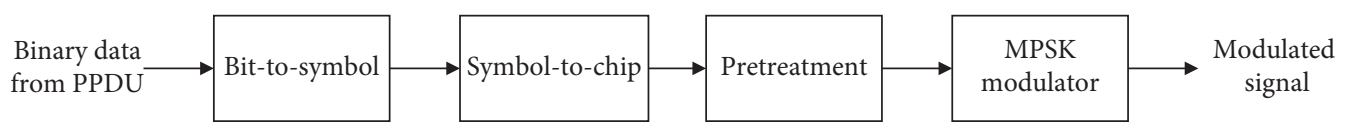

FIgURE 1: Data transmission process for MPSK physical layer in IEEE 802.15.4c.

After demapping, we can obtain the final detection result. This detection scheme is based on [23] but is different from [23]. The signal model in [23] only considers phase offset. In this work, we further considered CPO, spread spectrum, and slow Rayleigh channel. Therefore, we summarize the detailed process of the complete MSD program.

As shown in (3), based on an exhaustive search, 256 detection metrics need to be calculated for the full MSD even if we set the observation window length $N$ to 2 . This is clearly complexity-heavy. In order to make MSD easy for hardware implementation, we consider two simple strategies, which parallels Wilson's approach in [15]. First, we find the best and second-best decisions in each symbol position with the standard SBSD procedure characterized by (3) and freeze the global best decision. Second, for the remaining symbol position, only the best and second-best symbol decisions, not all the candidates, are jointly searched by the standard MSD procedure. Here, a qualitative explanation for this configuration is as follows. Apparently, the detection metric given in (3) can partly reflect the reliability of the decision result in each symbol position. Therefore, it is reasonable that the global best decision with the standard SBSD procedure characterized by (3) is the most reliable and can be frozen especially for high signal-to-noise ratio (SNR). Moreover, under high SNR, only searching the best and second-best symbol decision for the remaining symbol position is also feasible. In Section 6, we will further verify its rationality through quantitative simulations.

\section{The Proposed Detection Scheme}

For each symbol position, with the standard SBSD procedure characterized by (3), we can easily obtain two local metrics, that is, the best metric and second-best metric. Then, we froze the decision result corresponding to the most reliable symbol position, which is achieved by searching all the local best metric. For the remaining symbol position, the number of symbols to be searched is truncated. That is to say, only the symbols corresponding to the local best and second-best metrics are considered as the candidates. In this context, for observation window length $N=2$, we have reduced the number of the metrics given in (3) to be calculated from 256 to 2 . However, the simulation results in Section 6 show that the performance loss is very small. The specific implementation process is detailed as follows.

For the $i$ th block, the decision metric for each symbol position is first calculated as

$$
V_{x, y}=\left|w_{x, y}\right|^{2}, \quad 1 \leq y \leq 16 .
$$

Here, $w_{x, y}=\sum_{m=1}^{16} r_{x, m}^{\prime} s_{y, m}^{*}$, which is the complex crosscorrelation function.

Secondly, the best and the second-best metrics for the $n$th symbol in the $i$ th block can be given as follows:

$$
\begin{aligned}
& V_{N(i-1)+n, \hat{y}_{1}}=\underset{1 \leq \hat{y}_{1} \leq 16}{\arg \max }\left\{V_{N(i-1)+n, \hat{y}_{1}}\right\}, \quad i \geq 1, n=1,2, \ldots, N, \\
& V_{N(i-1)+n, \hat{y}_{2}}=\underset{1 \leq \hat{y}_{2} \leq 16, \hat{y}_{2}+\hat{y}_{1}}{\arg \max }\left\{V_{N(i-1)+n, \hat{y}_{2}}\right\}, \quad i \geq 1, n=1,2, \ldots, N,
\end{aligned}
$$

where $\hat{y}_{1}$ and $\hat{y}_{2}$, respectively, represent the estimated value of the index for the PN sequence corresponding to the best and second-best metrics of the $n$th symbol. For example, we can see that, as shown in Figure 2, a compensated baseband chip sequence $r_{1, m}^{\prime}$ passes through decision block 1 to generate decision set $\left\{V_{1,1}, V_{1,2}, \ldots, V_{1,16}\right\}$, and the best and second-best metrics in the decision set are recorded as $V_{1, \hat{y}_{1}}$ and $V_{1, \hat{y}_{2}}$, respectively.
Furthermore, find the global best metric, and freeze the detection result:

$$
\text { find } \widehat{n} \text { and } s_{\widehat{y}_{1}} \text { if } V_{N(i-1)+\widehat{n}_{n} \widehat{y}_{1}} \text { is maximum, }
$$

that is, let the detection result of the $\hat{n}$ th symbol be $\boldsymbol{s}_{\hat{y}_{1}}$. Figure 2 gives the implementation structure.

Finally, the data in the remaining $N-1$ symbol periods are jointly determined as follows:

$$
\text { find }\left\{\mathbf{s}_{\hat{y}_{k}}\right\} \text { for } n \neq \hat{n} \text { if }\left|w_{N(i-1)+\widehat{n}, \hat{y}_{1}}+\sum_{n \neq \widehat{n}} w_{N(i-1)+n, \hat{y}_{k}}\right|^{2}, \quad k \in\{1,2\} \text { is maximum. }
$$




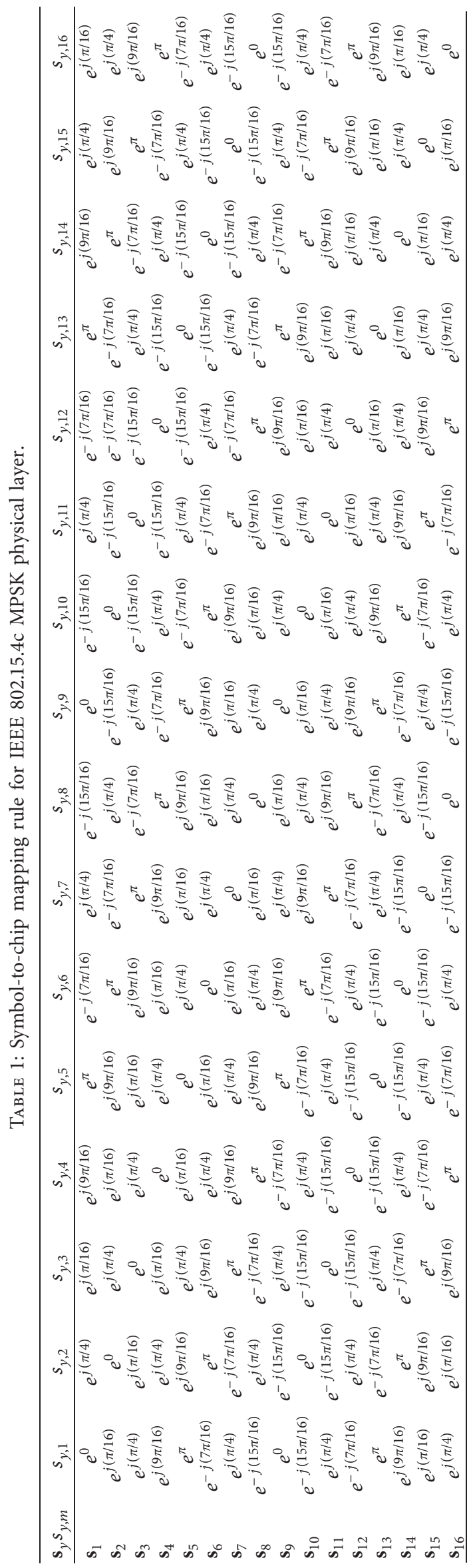




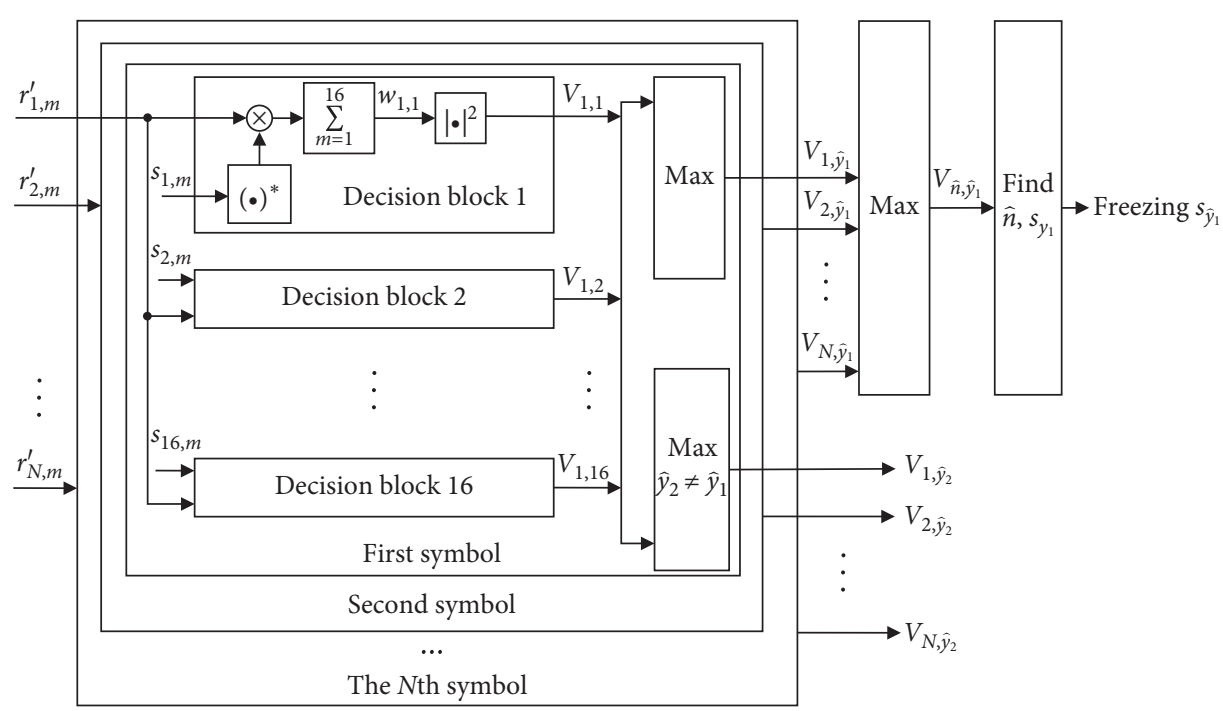

FIgURe 2: The structure of the first decision, where $i=1$, and the observation window length is $N$.

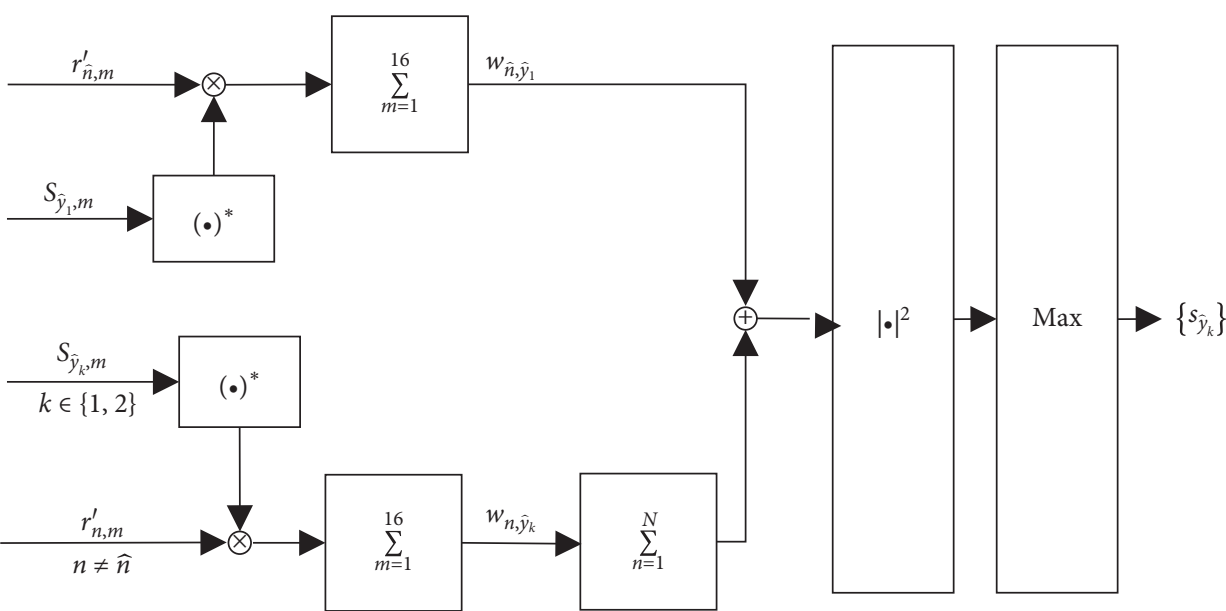

FIgure 3: The structure of the joint decision, where $i=1$, and the observation window length is $N$.

where $\hat{n}$ and $\hat{y}_{1}$ are given by (8). Figure 3 is a structural diagram of this joint decision.

Algorithm 1 introduces the detailed implementation step of proposed MSD scheme. For simple implementation, we only selected the most and second-most reliable symbols here. More metrics can also be involved, which, however, are complexity-intensive and not suitable for our purposes. In essence, when 16 metrics are selected, we arrive at the full MSD. Furthermore, the simulation results in Section 6.2 show that excellent performance has been exhibited even if we only equip the MSD scheme with the most and secondmost reliable metrics.

\section{Estimation Scheme}

Clearly, the chip sample $r_{x, m}$ in (1) is dependent on the transmitted chip symbol $s_{x, m}$, but this dependence can be eliminated if we follow the property $s_{x, m} s_{x, m}^{*}=1$ :

$$
G r_{x, m} \triangleq r_{x, m} s_{x, m}^{*}=h e^{j\left(m \omega T_{c}+\theta\right)}+\eta_{x, m} s_{x, m}^{*}, \quad 1 \leq x \leq P_{1}, 1 \leq m \leq M,
$$

where $P_{1}$ is the length of the preamble, $1 \leq P_{1} \leq P$, and $P=8$ is the maximum length. $\eta_{x, m} s_{x, m}^{*}$ is statistically equivalent to $\eta_{x . m}$. In this context, our purpose is to estimate $\omega T_{c}$ based on the sample observations given in (10).

Within $N$ symbol intervals, the normalized autocorrelation function of samples is as follows:

$$
Z(n)=\frac{1}{\left(P_{1}-n\right) L_{1}} \sum_{x=1}^{P_{1}} \sum_{m=2}^{L_{1}}\left(G r_{x, m} G r_{x, m-n}^{*}\right)=|h|^{2} e^{j \omega n T_{c}}+\eta_{n},
$$

where $L_{1}$ is the sample number of the preamble, and $2 \leq L_{1} \leq M . \eta_{n}$ represents the integrated noise. $n$ denotes the 
Input:

$r_{x, m}$ : baseband samples of the $x$ th bit $E[x]$

$J$ : PPDU payload length

$L_{1}$ : sample num ber of the $x$ th symbol in the preamble

$M$ : length of the PN sequence

$K$ : the maximum chip delay number

$N$ : observation window length

$P_{1}$ : preamble length

In order to simplify the detection process, $K$ is set 4 in this algorithm.

Output:

$\mathbf{s}_{\widehat{y}_{1}}$ and $\left\{\boldsymbol{s}_{\hat{y}_{k}}\right\}$ : detect the spread spectrum sequence of the actual data.

(1) initial $J=44, M=16, K=4, Z(n)=0, Q=0$

(2) $r_{x, m}$ to eliminate the influence of $s_{x, m}$

(3) for $x=1 ; x \leq P_{1} ; x++$ for

(4) for $m=2 ; m \leq M ; m++$ for

(5) $\quad \mathrm{Gr}_{x, m} \longleftarrow r_{x, m} s_{x, m}^{*}$

(6) end for

(7) end for

(8) for $n=1 ; n \leq K ; n++$ do

(9) for $x=1 ; x \leq P_{1} ; x++$ do

(10) for $m=2 ; m \leq L_{1} ; m++$ do

(11) $\quad Z(n) \longleftarrow Z(n)+G r_{x, m} G r_{x, m-n}^{*}$

(12) end for

(13) end for

(14) $Z(n) \longleftarrow Z(n) L_{1}\left(P_{1}-n\right)$

(15) $Q \longleftarrow Q+Z(n)$

(16) end for

(17) The quantization function of frequency offset estimator $g(Q) \leftarrow(2 / K+1) \arg (Q)$, where $\widehat{\varphi} \triangleq \omega T_{c}=g(Q)$

(18) for $x=1 ; x \leq P+(J / 4) ; x++$

(19) for $y=1 ; y \leq 16 ; y++$

(20) for $m=1 ; m \leq 16 ; m++$

$$
\begin{aligned}
& r_{x, m}^{\prime} \longleftarrow r_{x, m} e^{-j m \hat{\varphi}} \\
& w_{x, y} \longleftarrow w_{x, y}+r_{x, m}^{\prime} s_{y, m}^{*}
\end{aligned}
$$

$$
\text { end for }
$$$$
V_{x, y} \longleftarrow\left|w_{x, y}\right|^{2}
$$

(26) end for

(27) for $i=1 ; i \leq L / 4 N ; i++$

(28) for $n=1 ; n \leq N ; n++$

(29) for $\hat{y}_{1}=1 ; \hat{y}_{1} \leq 16 ; \hat{y}_{1}++$

(30) for $\widehat{y}_{2}=1 ; \hat{y}_{2} \leq 16 ; \hat{y}_{2}++$

(31)

(34)

(35)

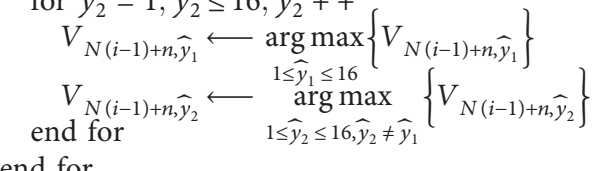

(36) end for

(37) find $\hat{n}$ and $s_{\hat{y}_{1}}$ if $V_{N(i-1)+\widehat{n}_{1}, \hat{y}_{1}}$ is maximum, let the judgment result of the $\hat{n}$ th symbol be $s_{\hat{y}_{1}}$

(38) find $\left\{\mathbf{s}_{\hat{y}_{k}}\right\}$ for $n \neq \hat{n}$ if $\left|w_{N(i-1)+\widehat{n}, \widehat{y}_{1}}+\sum_{n \neq \hat{n}} w_{N(i-1)+n, \hat{y}_{k}}\right|^{2}, k \in\{1,2\}$ is maximum

(39) return $\mathbf{s}_{\hat{y}_{1}}$ and $\left\{\mathbf{s}_{\hat{y}_{k}}\right\}$

Algorithm 1: The proposed detection algorithm.

number of chip delays, and $1 \leq n \leq K$. $K$ represents the maximum chip-delay number.

Following the idea in [24], a simple estimation scheme without phase unwrapping can be expressed as follows:

$$
\widehat{\varphi}=\widehat{\omega T c}=g(Q) \text {, }
$$

where the quantization function $g(Q)$ is

$$
g(Q)=\frac{2}{K+1} \arg (Q)
$$

where $Q=\sum_{n=1}^{K} Z(n)$. The structure of this estimator is shown in Figure 4. 


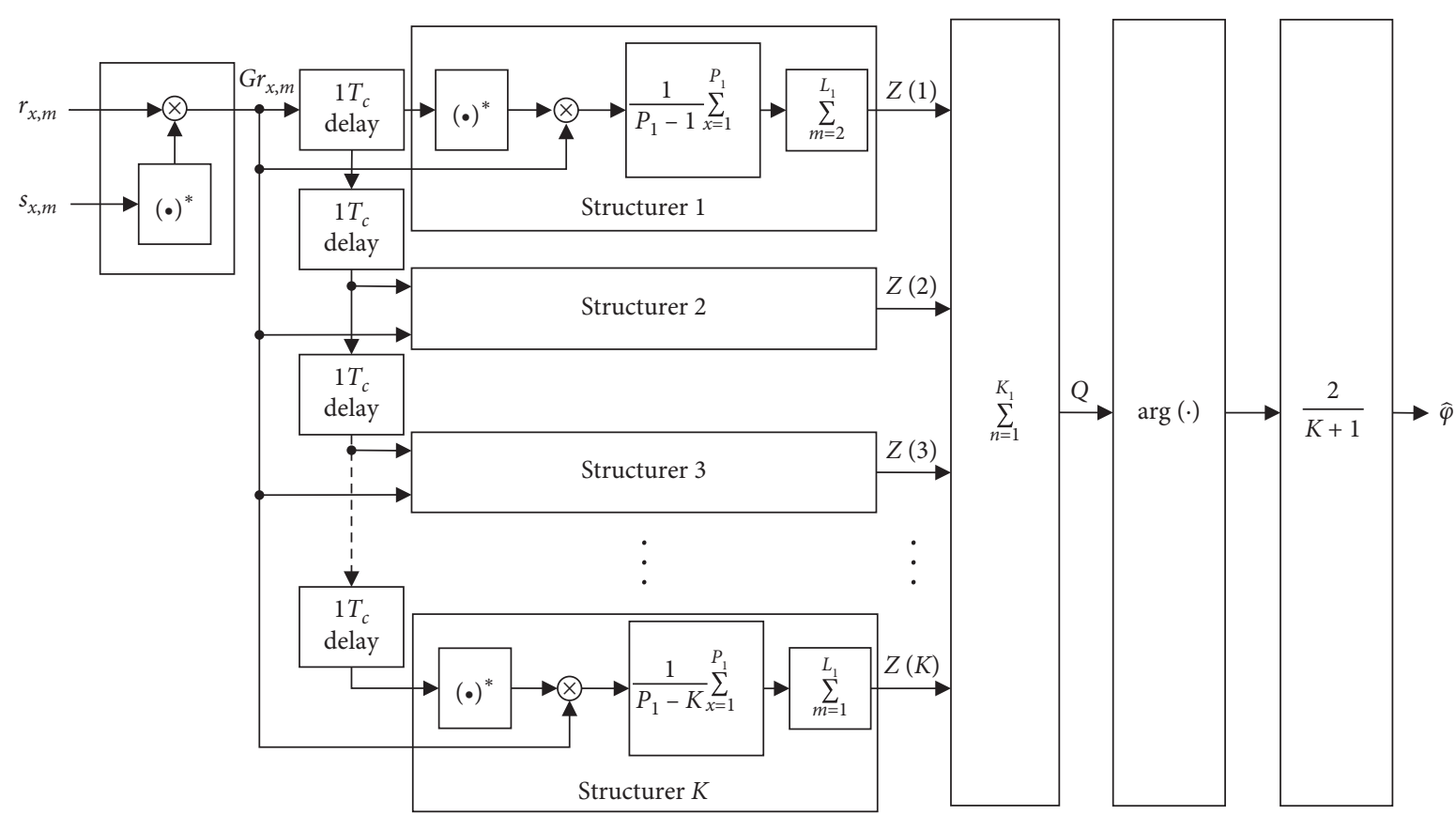

FIgURE 4: The structure diagram of frequency offset estimator.

TABle 2: Parameters used in simulations.

\begin{tabular}{lc}
\hline Parameter & Detailed description \\
\hline Channel condition & Slow fading or pure AWGN \\
Power of the complex AWGN & N/SNR \\
Power of Rayleigh fading channel & MSD \\
Detection scheme & Precompensation \\
Compensation scheme & Perfect \\
Timing synchronization & MPSK \\
Data modulation & 16 -ary orthogonal \\
Symbols & 176 \\
PPDU payload length (bits) & 16 \\
Spreading factor & 1 \\
Chip rate $($ Mchip/s) & 250 \\
Binary data rate $(\mathrm{kb} / \mathrm{s})$ & 786 \\
Carrier frequency $(\mathrm{MHz})$ & Symmetrical triangular distribution in $(-80,80)$ \\
CFO $f$ (ppm) & Uniform distribution in $(-\pi, \pi)$ \\
CPO $\theta$ (rads) & 16 \\
PN length $M$ & 8 \\
Preamble length $P_{1}$ & 16 \\
Sample number $L_{1}$ & \\
\hline
\end{tabular}




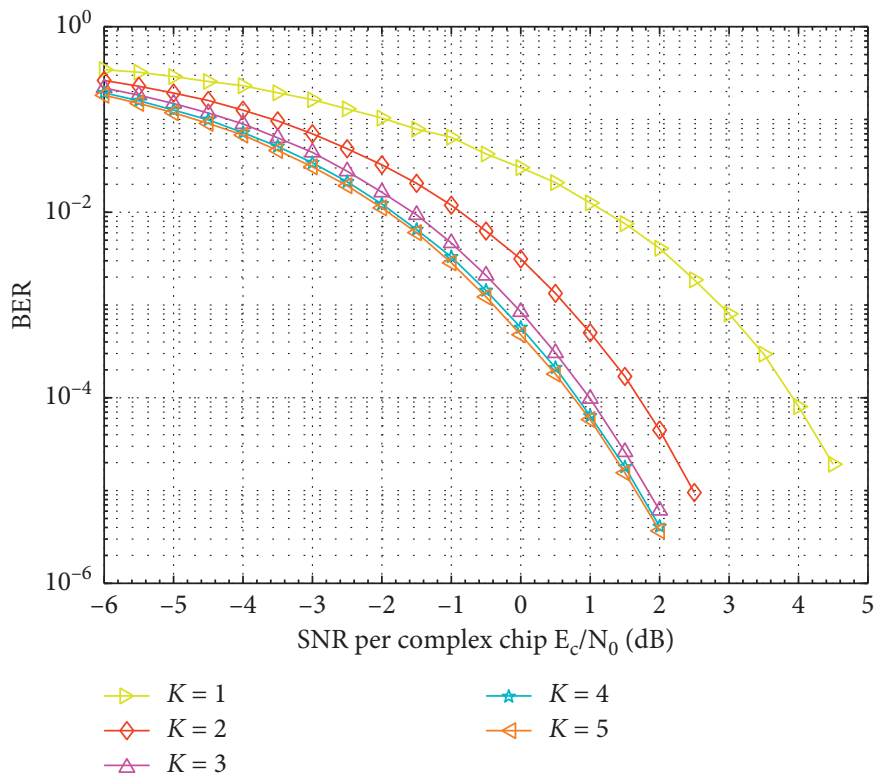

(a)

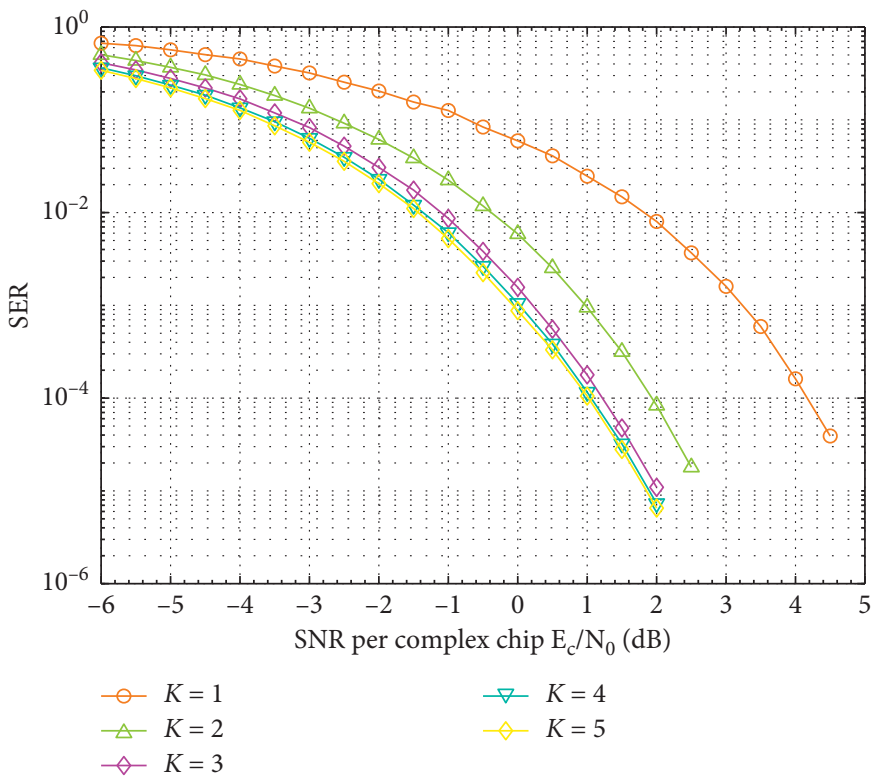

(b)

FIgUre 5: Continued. 


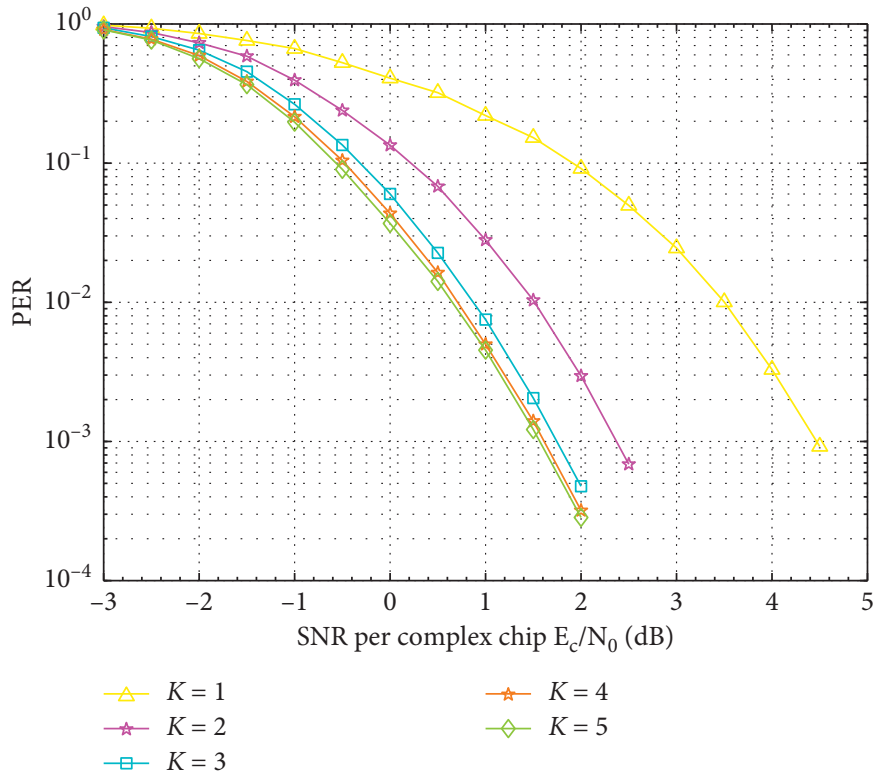

(c)

FIGURE 5: In a pure AWGN channel, the effect of parameter $K$ on the performance of the full estimator in $(16), N=2$. (a) BER performance; (b) SER performance; (c) PER performance.

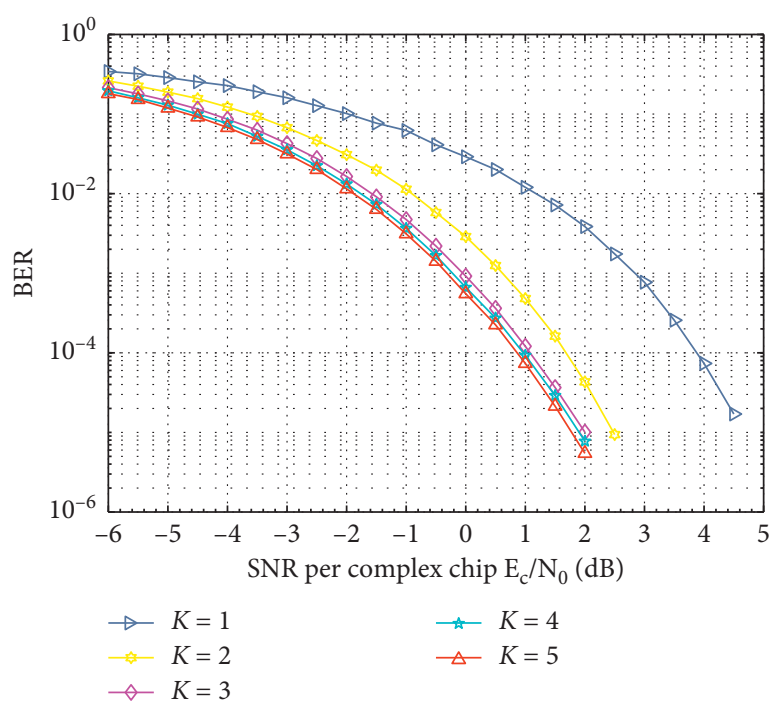

(a)

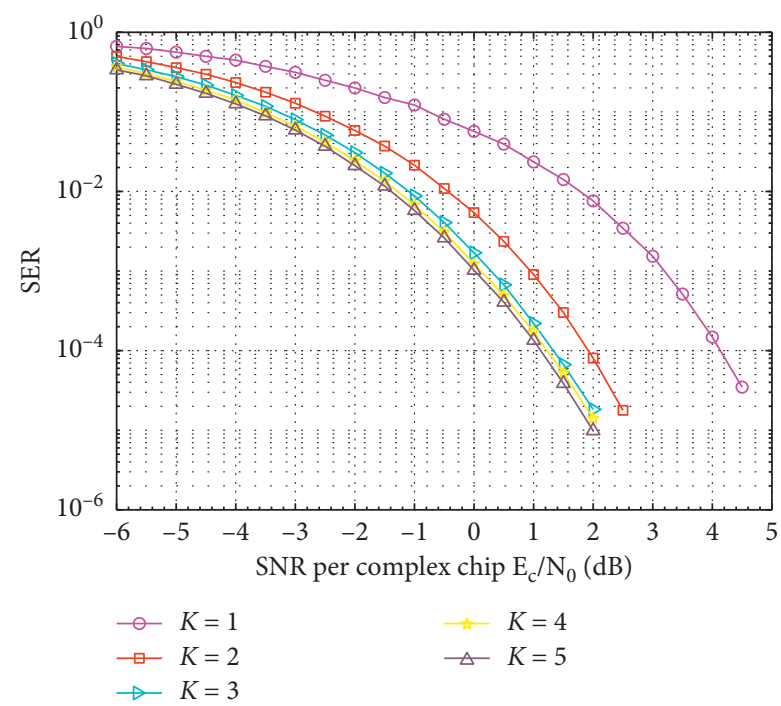

(b)

Figure 6: Continued. 


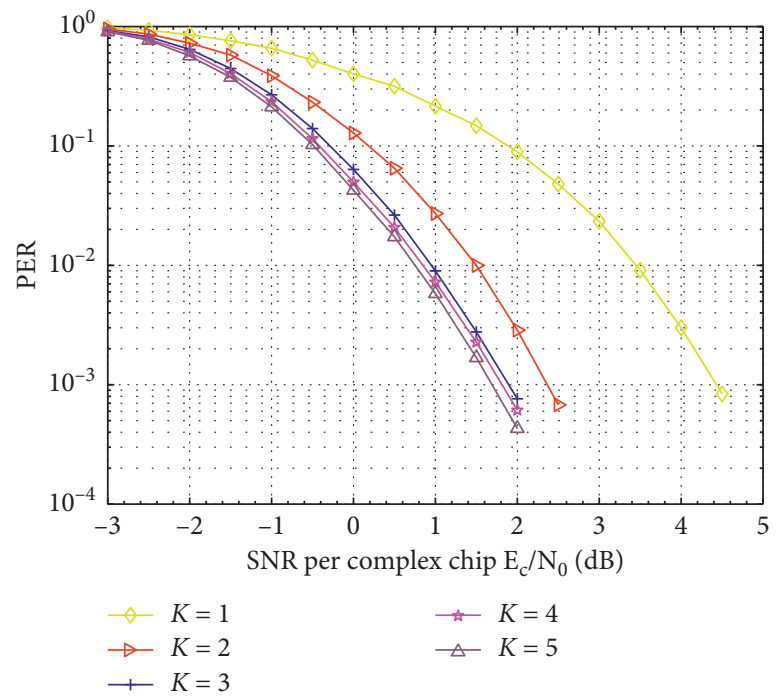

(c)

FIGURE 6: In a pure AWGN channel, the effect of parameter $K$ on the performance of the simplified estimator in (15), $N=2$. (a) BER performance; (b) SER performance; (c) PER performance.

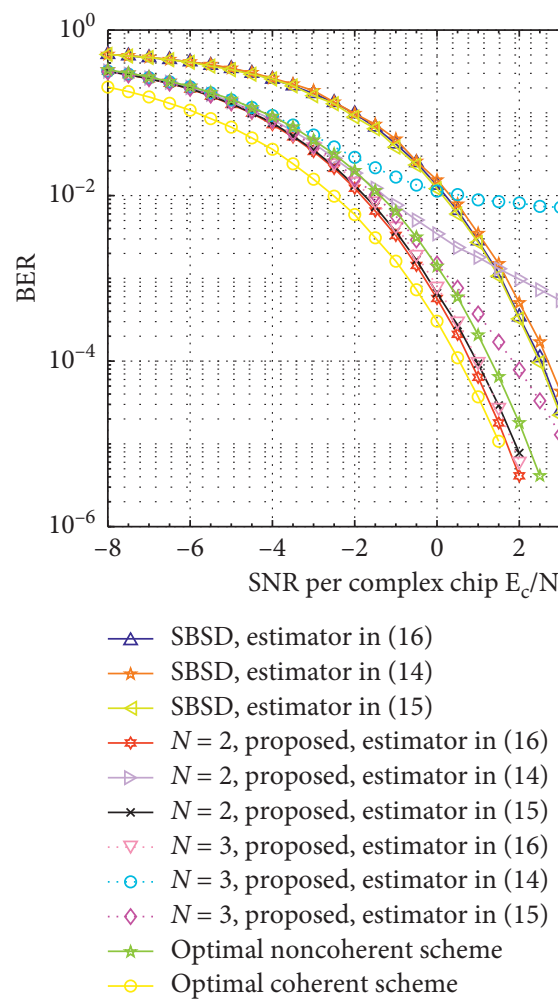

(a)

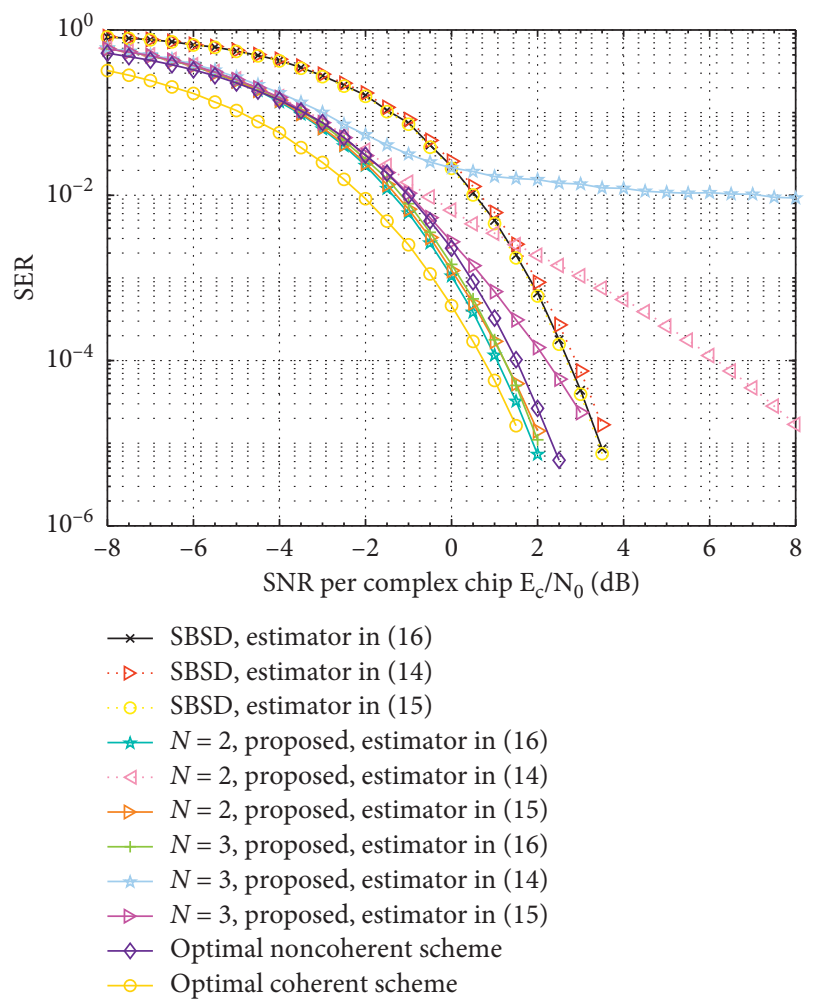

(b)

Figure 7: Continued. 


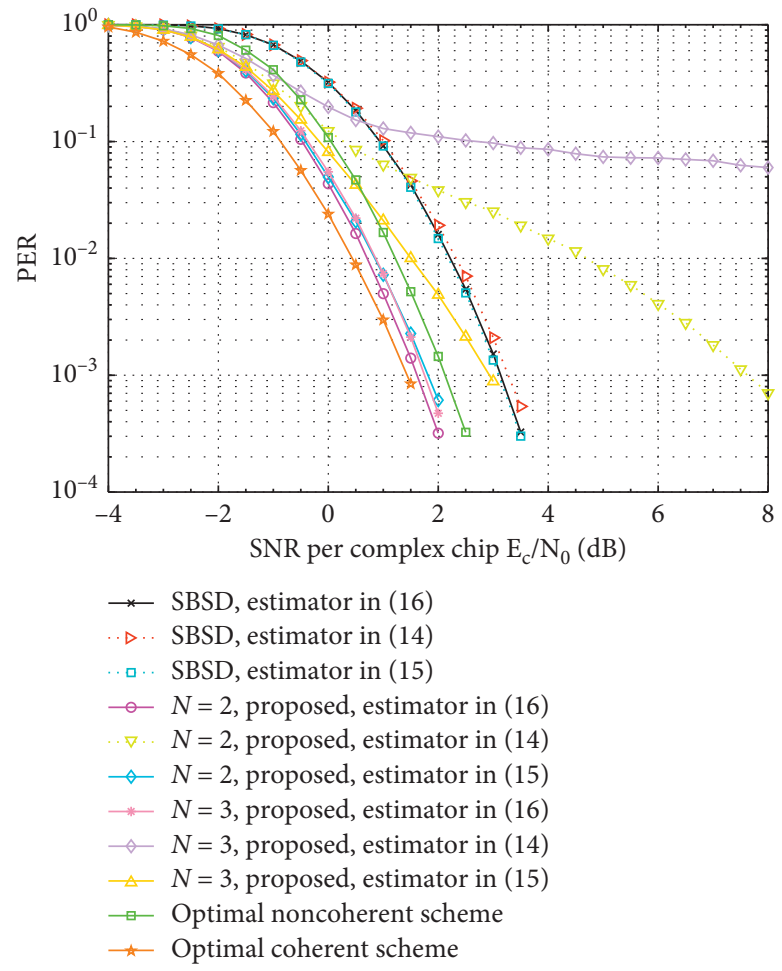

(c)

FIGURE 7: Performance comparison of different receivers in pure AWGN channel. (a) BER performance; (b) SER performance; (c) PER performance.
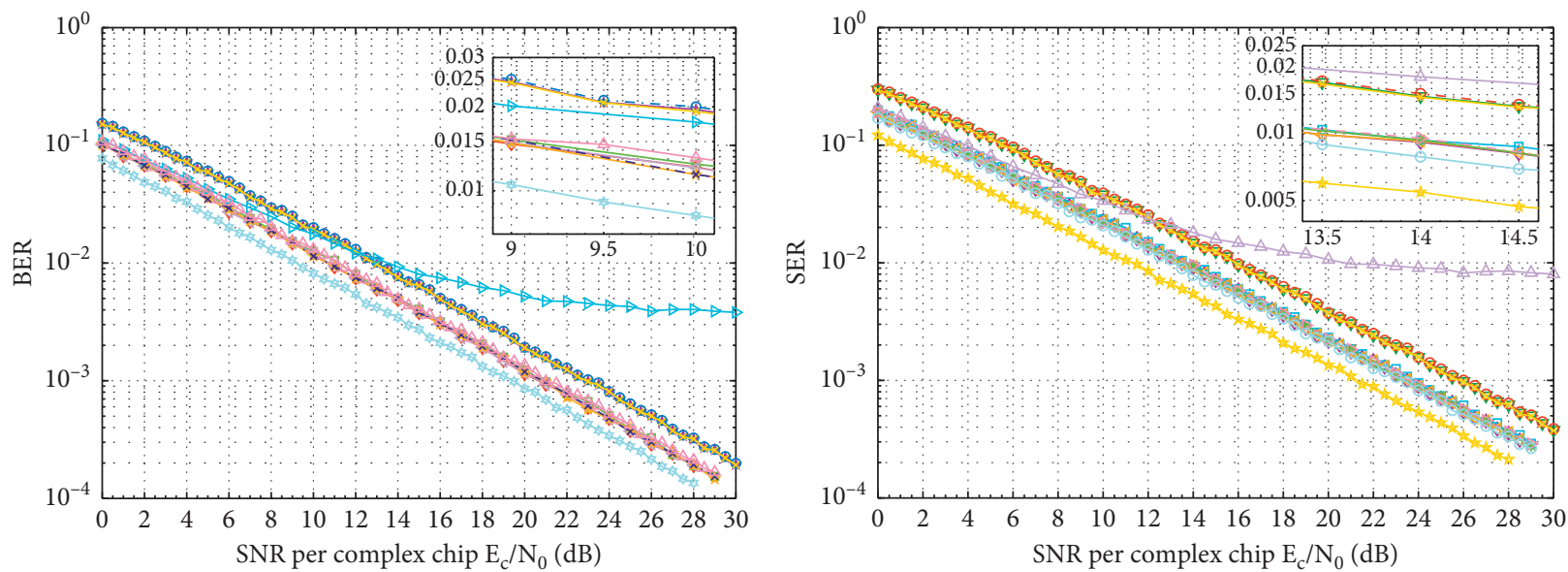

\footnotetext{
- SBSD, estimator in (16)

- - SBSD, estimator in (14)

* SBSD, estimator in (15)

$\leadsto N=2$, proposed, estimator in (16)

$\because \quad N=2$, proposed, estimator in (14)

$\dashv N=2$, proposed, estimator in (15)

\# $N=3$, proposed, estimator in (16)

$\rightarrow N=3$, proposed, estimator in (14)

- $-x-N=3$, proposed, estimator in (15)

$\triangle$ Optimal noncoherent scheme

$\rightarrow$ Optimal coherent scheme
}

$$
\begin{aligned}
& \rightarrow \text { SBSD, estimator in (16) } \\
& \rightarrow-\text { SBSD, estimator in (14) } \\
& \rightarrow \text { SBSD, estimator in (15) } \\
& \rightarrow \quad N=2 \text {, proposed, estimator in (16) } \\
& \square \quad N=2 \text {, proposed, estimator in (14) } \\
& \neg N=2 \text {, proposed, estimator in (15) } \\
& \rightarrow \quad N=3 \text {, proposed, estimator in (16) } \\
& \rightarrow \quad N=3 \text {, proposed, estimator in (14) } \\
& \rightarrow-N=3 \text {, proposed, estimator in (15) } \\
& \rightarrow \text { Optimal noncoherent scheme } \\
& \rightarrow \text { Optimal coherent scheme }
\end{aligned}
$$

(b)

Figure 8: Continued. 


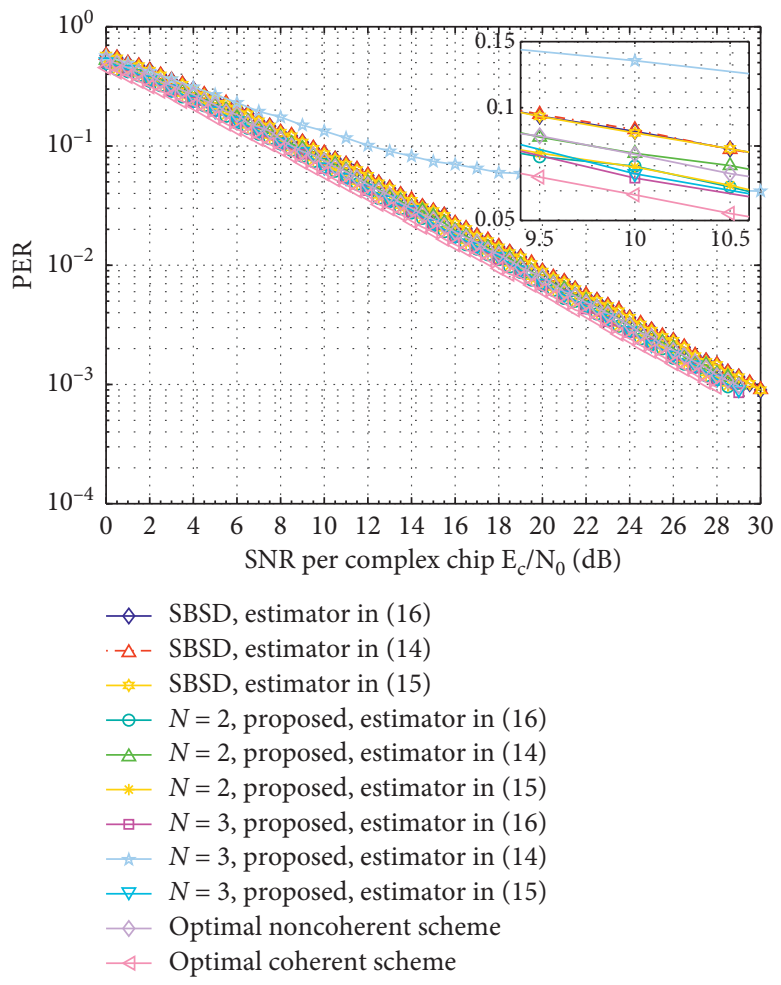

(c)

FIGURE 8: Performance comparison of different receivers in slow fading Rayleigh channel. (a) BER performance; (b) SER performance; (c) PER performance.

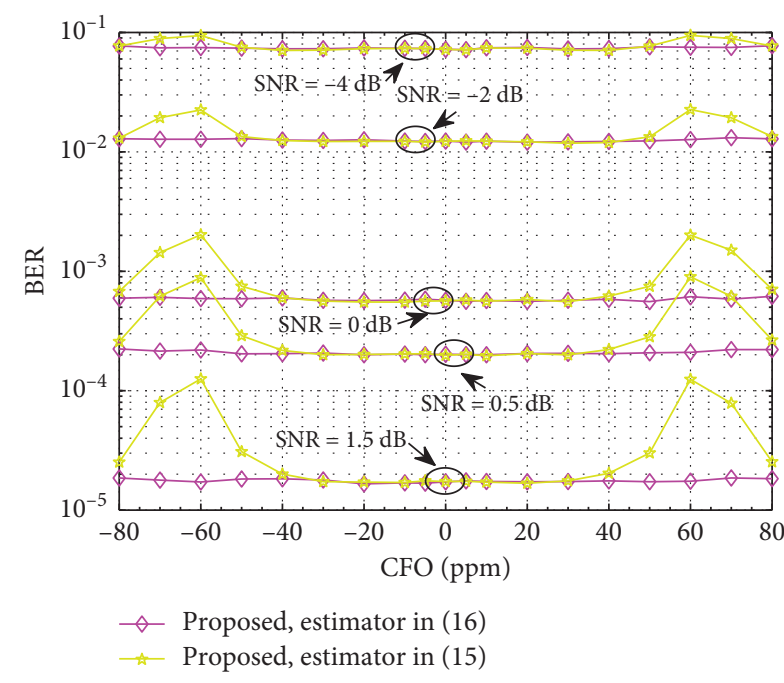

(a)

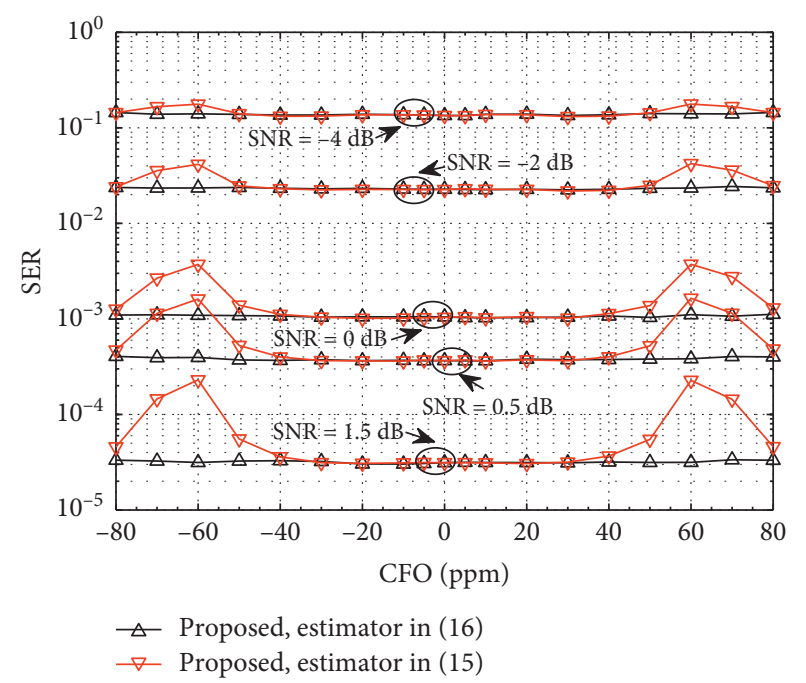

(b)

FIgURE 9: Continued. 


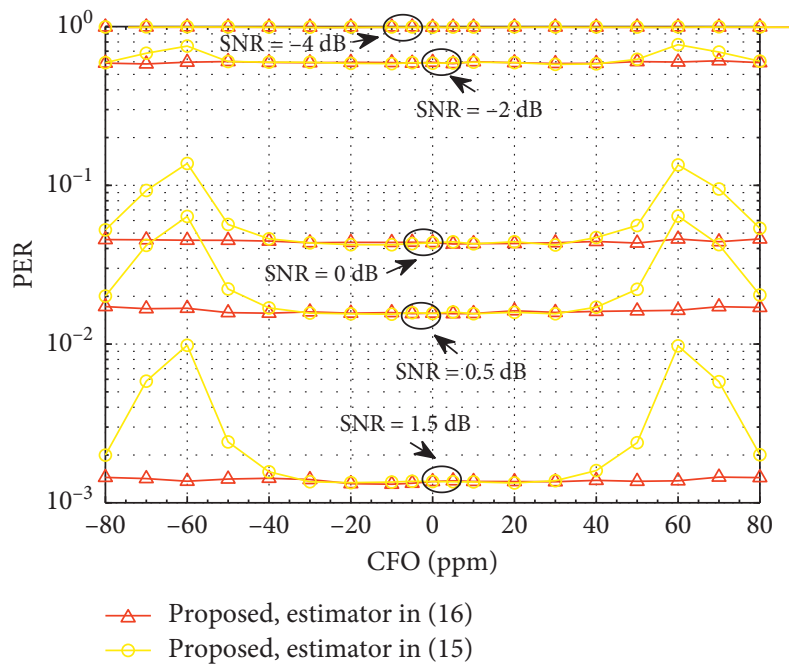

(c)

FIGURE 9: Detection performance comparisons of the proposed scheme under various estimators versus CFO over pure AWGN channel, $N=2$. (a) BER performance; (b) SER performance; (c) PER performance.

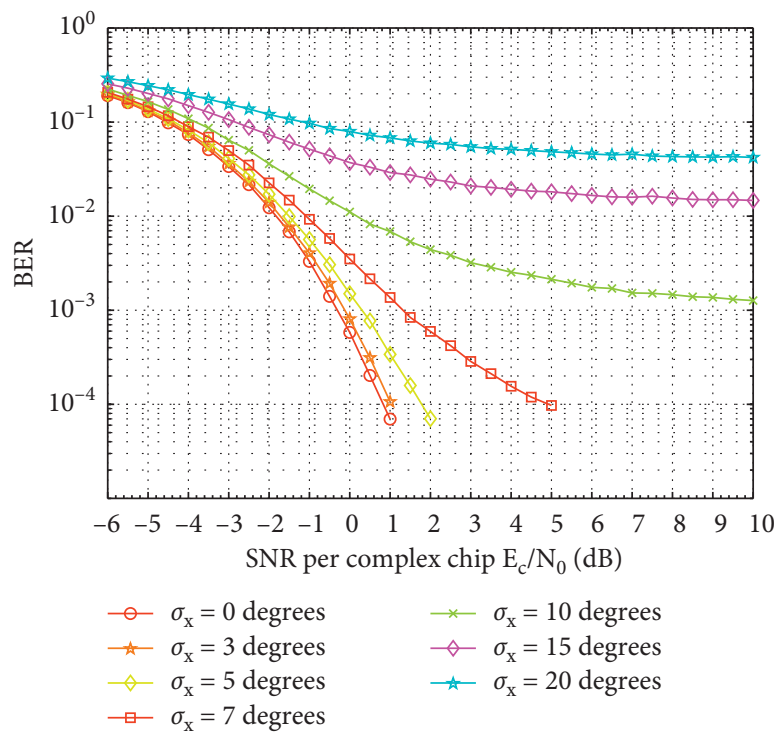

(a)

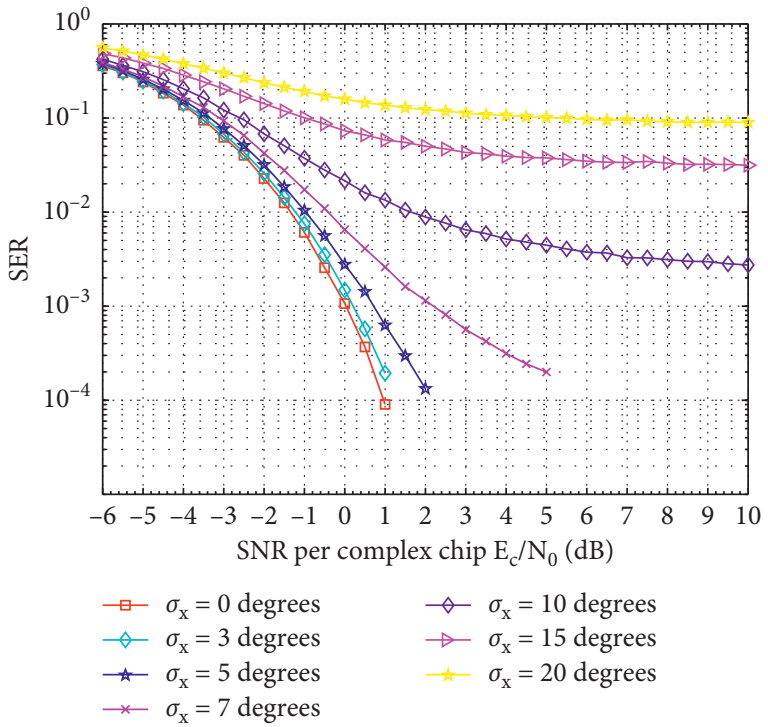

(b)

Figure 10: Continued. 


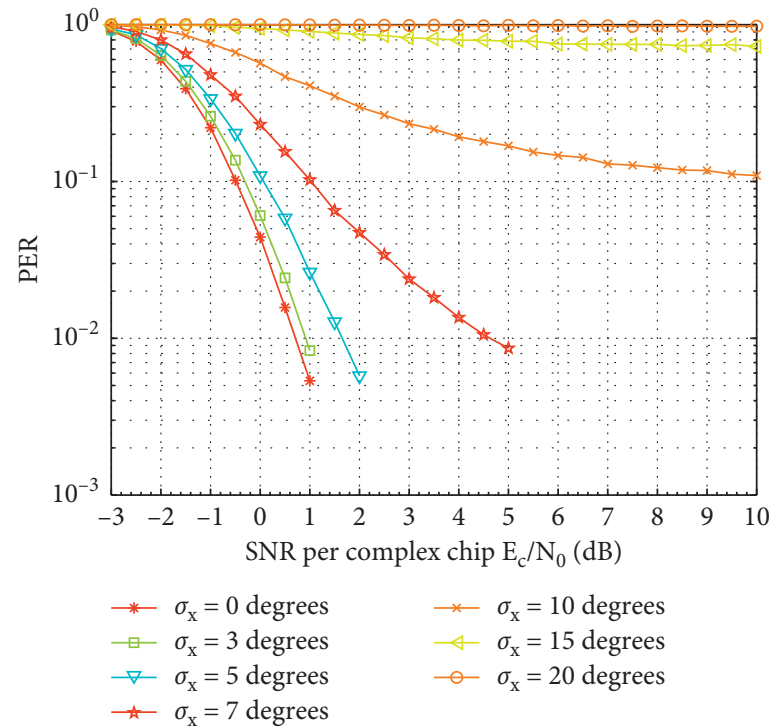

(c)

FIGURE 10: In the pure AWGN channel, the proposed scheme is compared with the detection performance of dynamic CPO under the full estimator in (16); $N=2$. (a) BER performance; (b) SER performance; (c) PER performance.

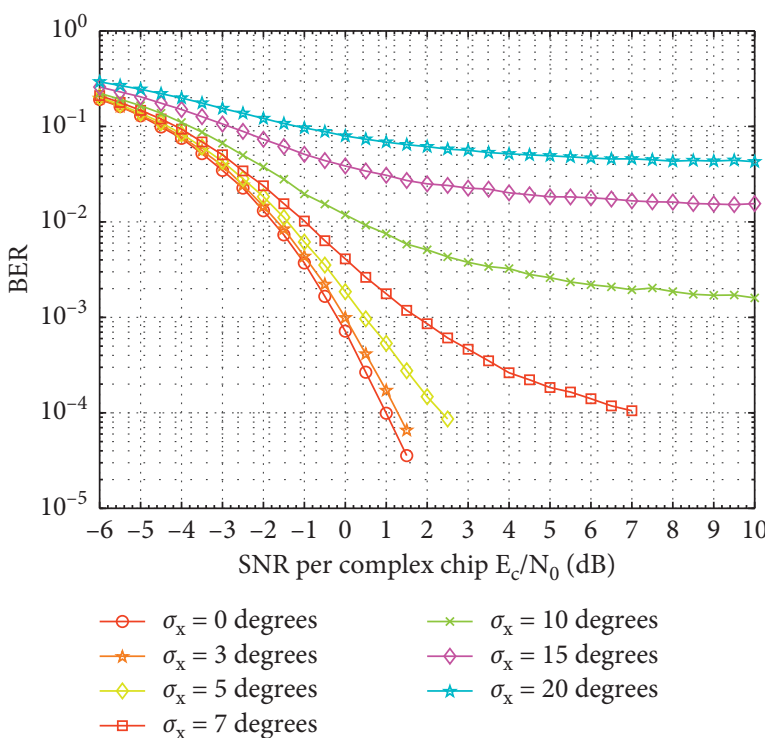

(a)

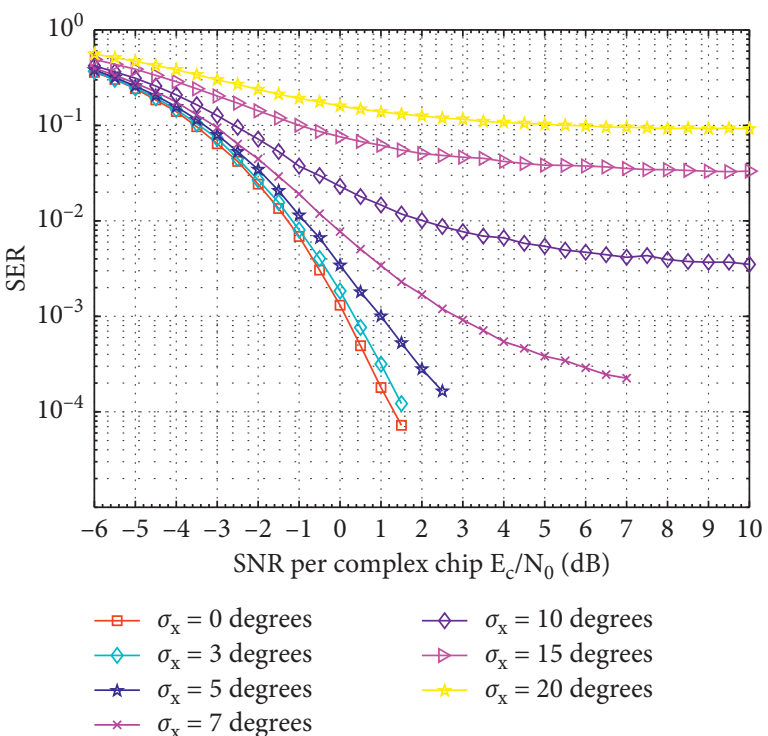

(b)

FIgURE 11: Continued. 


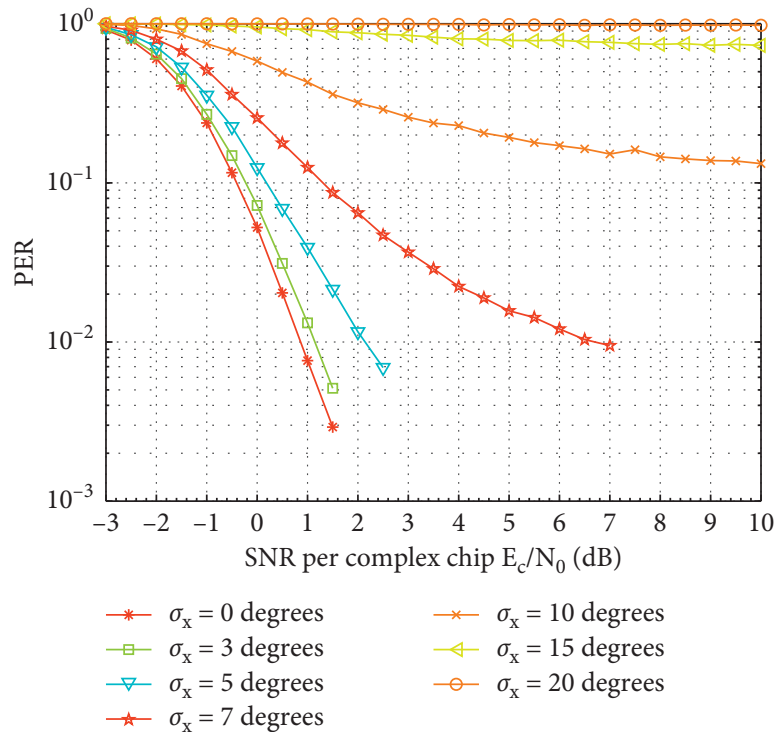

(c)

FIGURE 11: In the pure AWGN channel, the proposed scheme is compared with the detection performance of dynamic CPO under simplified estimator in (15); $N=2$. (a) BER performance; (b) SER performance; (c) PER performance.

It is apparent from (13) and Figure 4 that the estimation process involves complex inverse tangent operation.
According to our previous work [21, 25-27], two simplified estimation schemes can be obtained:

$$
\begin{aligned}
& \int \frac{2}{K+1} \frac{\operatorname{Im}(Q)}{\operatorname{Re}(Q)}, \quad \text { if } \operatorname{Re}(Q)>0 \text { and }|\operatorname{Re}(Q)| \geq|\operatorname{Im}(Q)| \\
& \widehat{\varphi} \approx \begin{cases}\frac{2}{K+1}\left(\frac{\pi}{2}-\frac{\operatorname{Re}(Q)}{\operatorname{Im}(Q)}\right), & \text { if } \operatorname{Im}(Q)>0 \text { and }|\operatorname{Re}(Q)|<|\operatorname{Im}(Q)|, \\
\frac{2}{K+1}\left(-\pi+\frac{\operatorname{Im}(Q)}{\operatorname{Re}(Q)}\right), & \text { if } \operatorname{Re}(Q)<0 \text { and }|\operatorname{Re}(Q)| \geq|\operatorname{Im}(Q)|, \\
\frac{2}{K+1}\left(-\frac{\pi}{2}-\frac{\operatorname{Re}(Q)}{\operatorname{Im}(Q)}\right), & \text { if } \operatorname{Im}(Q)<0 \text { and }|\operatorname{Re}(Q)|<|\operatorname{Im}(Q)|,\end{cases} \\
& \widehat{\varphi} \approx \begin{cases}\frac{2}{K+1} \frac{\operatorname{Im}(Q)}{\sqrt{\operatorname{Re}^{2}(Q)+\operatorname{Im}^{2}(Q)},} \quad \text { if } \operatorname{Re}(Q)>0 \text { and }|\operatorname{Re}(Q)| \geq|\operatorname{Im}(Q)|, \\
\frac{2}{K+1}\left(\frac{\pi}{2}-\frac{\operatorname{Re}(Q)}{\sqrt{\operatorname{Re}^{2}(Q)+\operatorname{Im}^{2}(Q)}}\right), & \text { if } \operatorname{Im}(Q)>0 \text { and }|\operatorname{Re}(Q)|<|\operatorname{Im}(Q)|, \\
\frac{2}{K+1}\left(-\pi-\frac{\operatorname{Im}(Q)}{\left.\sqrt{\operatorname{Re}^{2}(Q)+\operatorname{Im}^{2}(Q)}\right),}\right. & \text { if } \operatorname{Re}(Q)<0 \text { and }|\operatorname{Re}(Q)| \geq|\operatorname{Im}(Q)|, \\
\frac{2}{K+1}\left(-\frac{\pi}{2}+\frac{\operatorname{Re}(Q)}{\left.\sqrt{\operatorname{Re}^{2}(Q)+\operatorname{Im}^{2}(Q)}\right),}\right. & \text { if } \operatorname{Im}(Q)<0 \text { and }|\operatorname{Re}(Q)|<|\operatorname{Im}(Q)| .\end{cases}
\end{aligned}
$$


TABLE 3: On the pure AWGN channel, the implementation complexity of the proposed receiver and the full form receiver and $N=2$.

\begin{tabular}{lccc}
\hline Scheme & $(\cdot)(\cdot)^{*}$ & $(\cdot)+(\cdot)$ & $|\cdot|^{2}$ \\
\hline Proposed & 576 & 573 & 34 \\
Full complexity & 8192 & 8064 & 256 \\
\hline
\end{tabular}

For integrity of this work, we also give the full estimation scheme here.

$$
\widehat{\varphi}= \begin{cases}\frac{2}{K+1} \tan ^{-1}\left(\frac{\operatorname{Im}(Q)}{\operatorname{Re}(Q)}\right), \quad \text { if } \operatorname{Re}(Q)>0 \text { and }|\operatorname{Re}(Q)| \geq|\operatorname{Im}(Q)|, \\ \frac{2}{K+1}\left(\frac{\pi}{2}-\tan ^{-1}\left(\frac{\operatorname{Re}(Q)}{\operatorname{Im}(Q)}\right)\right), & \text { if } \operatorname{Im}(Q)>0 \text { and }|\operatorname{Re}(Q)|<|\operatorname{Im}(Q)|, \\ \frac{2}{K+1}\left(-\pi+\tan ^{-1}\left(\frac{\operatorname{Im}(Q)}{\operatorname{Re}(Q)}\right)\right), & \text { if } \operatorname{Re}(Q)<0 \text { and }|\operatorname{Re}(Q)| \geq|\operatorname{Im}(Q)| \\ \frac{2}{K+1}\left(-\frac{\pi}{2}-\tan ^{-1}\left(\frac{\operatorname{Re}(Q)}{\operatorname{Im}(Q)}\right)\right), & \text { if } \operatorname{Im}(Q)<0 \text { and }|\operatorname{Re}(Q)|<|\operatorname{Im}(Q)|\end{cases}
$$

A data-aided detection scheme is considered for simple implementation. However, the non-data-aided detection method can also be applied. This follows from the fact that the modulated data within the sample chip can be easily wiped out with the aid of any PN code using cross-correlation operation. Then, the CFOE can be easily estimated and compensated. At present, there are many other CFO estimation schemes [28-31]. These schemes involve complex mathematical operations, such as the logarithmic operation, the exponential operation, and the trigonometric operation. Our scheme further simplifies these complex operations, as shown in (14) and (15).

\section{Simulation Result}

In this section, we evaluate the bit error rate (BER), symbol error rate (SER), and packet error rate (PER) performance of various detection schemes. Note that, in the simulation, the PPDU payload length is set to 22 bytes. We choose the maximum in $780 \mathrm{MHz}$ frequency band as the carrier frequency, that is, $786 \mathrm{MHz}$. The detailed simulation parameters are shown in Table 2.

6.1. Influence of Maximum Chip-Delay Number $K$ on Detection Performance. The performance of IEEE 802.15.4c MPSK receiver can be improved by introducing a compatible maximum chip-delay number $K$. In slow fading Rayleigh channel and the pure AWGN channel with different $K$, we compared the BER, SER, and PER of our proposed MSD scheme with the full estimator in (16) and the simplified estimator in (15).

It can be seen from Figures 5 and 6 that when the maximum chip-delay number $K$ increases from 1 to 5 , the BER, SER, and PER performance can improved under the pure AWGN channel. In particular, as depicted in Figure 5(c), when PER $=1 \times 10^{-3}$, as $K$ increases from 1 to 2 , the SNR gain is approximately $2.2 \mathrm{~dB}$; when $K$ increases from 2 to 3 , the SNR gain is about $0.5 \mathrm{~dB}$; when $K$ increases from 3 to 4 , the SNR gain is about $0.1 \mathrm{~dB}$. Furthermore, $K=$ 3 is sufficient to meet the performance requirements of the receiver in pure AWGN channel [9]. Also, the improvement is so small when the maximum chip-delay number ranges from 4 to 5 , so we set the maximum chip-delay number to be 4 , that is, $K=4$.

6.2. Detection Performance Comparison. The BER, SER, and PER results for various detection schemes under pure AWGN channel and slow fading Rayleigh channel are, respectively, shown in Figures 7 and 8. In theory, the full MSD scheme is extremely close to the optimal coherent detection with the increase of the observation window length $N$. The implementation of the full MSD scheme is too complex. For the convenience of comparison, we use the optimal coherent detection to replace the simulation results of the full MSD scheme. We take the optimal coherent detection as the lowest bound.

As shown in Figure 7, when $N=2$, the simplified estimation in (14) would lead to serious error floor. This is 
caused by the continuous accumulation of larger estimation errors in (3). However, when the full estimation in (16) and the simplified estimation in (15) are used, the detection performance is excellent. There is a little gap between those and the optimal coherent detection, especially at high SNR. Furthermore, when we have $1 \times 10^{-3}$, compared with the SBSD method, the proposed scheme can achieve gain about $1.6 \mathrm{~dB}$.

When $N$ is increased from 2 to 3 , the performance of the proposed detection schemes decreases. This is because when $N=3$, the error caused by the estimation scheme introduces a large accumulation in (3), and a mismatch is then observed between the estimator and the detector. In conclusion, the estimation scheme in this paper is especially suitable for the detection scheme, wherein the observation window length $N$ is set to be 2 . Moreover, when $N=2$, the performance gap between our proposed scheme and the optimal coherent detection is so small, and there is almost no more room for improvement. Therefore, we choose $N$ as 2 in the subsequent simulations. In addition, as shown in Figure 8, we can draw similar conclusions under slow fading Rayleigh channel, which, however, is not illustrated here.

\subsection{Frequency Offset Robustness of the Proposed Scheme in} Pure AWGNChannel. In the pure AWGN channel, we show the BER, SER, and PER performance results with different estimation schemes in Figure 9. CFO $f$ obeys a symmetry triangular distribution at $(-80,+80) \mathrm{ppm}$. The results of the full estimation in (16) are used as a benchmark. As shown in Figure 9, for the simplified estimation in (15), the detection performance is good for CFO between +60 and $-60 \mathrm{ppm}$. However, the performance fluctuates when the CFO is greater than $+60 \mathrm{ppm}$ or less than $-60 \mathrm{ppm}$. In addition, the performance fluctuation increases with the increase of SNR. However, according to the CFO probability distribution characteristic, the probability that the absolute value of CFO exceeds 60 is 0.0625 , which is very small. Thus, the proposed detection scheme is not sensitive to frequency offset.

6.4. CPO Robustness under Pure AWGN Channel. In this part, we study the detection performance of the proposed receiver in pure AWGN channel with changing carrier phase, where $N=2$. In Figures 10 and 11, the proposed scheme is robust to dynamic phase jitter. The phase $\theta$ is modeled as a Wiener process, wherein its initial value is uniformly chosen from $(-\pi, \pi)$. As shown in Figures 10 and 11 , the proposed scheme is robust to dynamic phase jitter. The performance of the proposed receiver does not significantly degrade if we increase the standard deviation of jitter from $0^{\circ}$ to $3^{\circ}$. In addition, an irreducible error floor is observed for the estimators given in (15) and (16) with the increase of SNR.

6.5. Complexity Analysis. We compare the implementation complexity of various detection schemes in pure AWGN channels. It is assumed that the full MSD and the proposed detection scheme are equipped with the same estimator. The

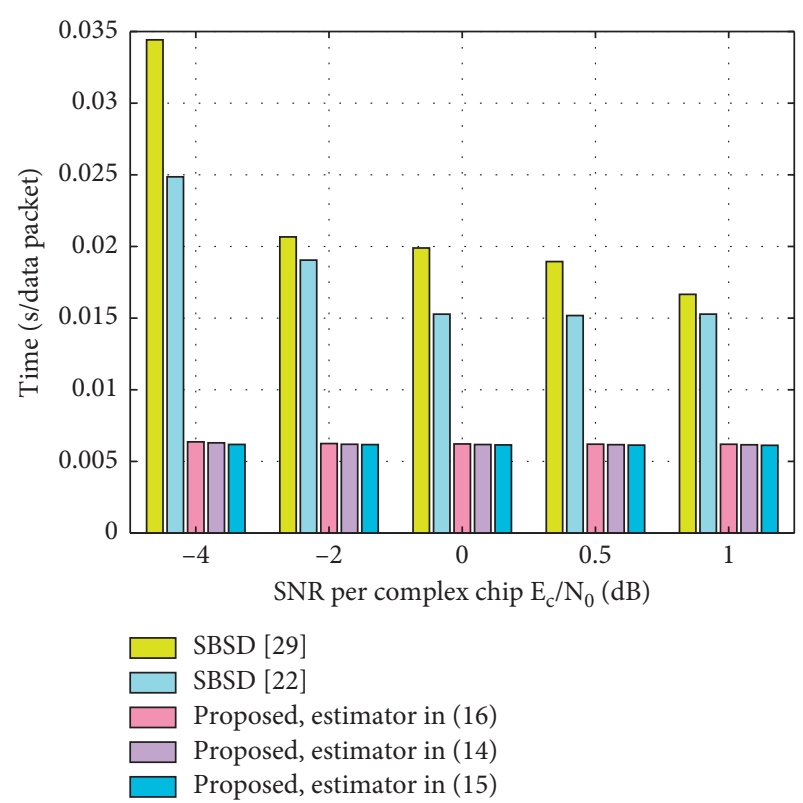

Figure 12: Average running time for different detection schemes in pure AWGN channel; $N=2$.

discrepancy in receiver implementation complexity is determined by the metrics given in (3), (5), and (9). Note that we set $J, L_{1}$, and $P_{1}$ to the maximum, that is, $J=44, L_{1}=16$, and $P_{1}=8$. The structure block diagram of multiplication operation is shown in Figure 3 of [32]. Complex addition is the addition of two complex numbers. It is assumed that a comparison operation is equivalent to an addition operation. As shown in Table 3, our proposed detection scheme only requires 576 complex multiplications, 573 complex additions, and 34 modular squaring operations. The full MSD given in Section 3 requires 8192 complex multiplications, 8064 complex additions, and 256 modular squaring operations. Obviously, compared with the full MSD, the complexity of our scheme is extremely reduced.

In addition, the average running time can also partly reflect the implementation complexity. Specifically, for different detection schemes, we develop various simulations by running enough number of transmission frames. In fact, $10^{5}$ frames of data are implemented, and the average running time is achieved. Surprisingly, as shown in Figure 12, when the SNR is $-4 \mathrm{~dB}$, the average running time for the traditional SBSD developed from [23] is 4 times as much as that of our proposed MSD scheme. Furthermore, as for the SBSD developed from [33], the average running time is 5.5 times as much as that of our MSD scheme.

\section{Conclusions}

In this paper, a simple but reliable MSD scheme for IEEE 802.15.4c MPSK receiver has been proposed, wherein the CFO has been estimated and compensated by using preamble assisted method. Experimental results showed that our detection performance can meet the requirements of WSN with only four maximum chip delays. In addition, when the standard deviation of the phase jitter is as high as 
$3^{\circ}$, the performance does not significantly decrease. Finally, compared with the full MSD scheme, our improved scheme is more attractive in terms of complexity. Therefore, the research results of this paper have a positive role in promoting the engineering application of the IoT in the field of new smart city.

In order to avoid the channel and CFO estimation, double-differential modulation is famously used as shown in [34-37]. This idea can be directly borrowed and implemented in our detection scheme for further complexity reduction. Note, however, that more performance loss is exhibited in this case.

\section{Data Availability}

All of the underlying data in this manuscript are available from the corresponding author upon request.

\section{Conflicts of Interest}

The authors declare that there are no conflicts of interest.

\section{Acknowledgments}

This work was partially supported by the National Natural Science Foundation of China (61701172, 41605122, 61701059, 61701062, 61801170, and 61772175), the Key Laboratory of Middle Atmosphere and Global Environment Observation (LAGEO-2021-04), the Natural Science Foundation of Henan Province (162300410097 and 162300410096), the Scientific and Technological Innovation Team of Colleges and Universities in Henan Province (20IRTSTHN018), Postdoctoral Science Foundation of University of Electronic Science and Technology of China (Y02006023601721), the Program for Everest Scholar Talents Development in Tibet University, and the Program for Science and Technology Innovation Talents in the University of Henan Province (Educational Committee) (17HASTIT025).

\section{References}

[1] M.-D. González-Zamar, E. Abad-Segura, E. Vázquez-Cano, and E. López-Meneses, "IoT technology applications-based smart cities: research analysis," Electronics, vol. 9, no. 8, p. 36, 2020.

[2] H. Habibzadeh, T. Soyata, B. Kantarci, A. Boukerche, and C. Kaptan, "Sensing, communication and security planes: a new challenge for a smart city system design," Computer Networks, vol. 144, pp. 163-200, 2018.

[3] G. Zhang, H. Wen, J. Pu, and J. Tang, "Build-in wiretap channel I with feedback and LDPC codes by soft decision decoding," IET Communications, vol. 11, no. 11, pp. 1808-1814, 2017.

[4] X. Li, M. Zhao, Y. Liu et al., "Secrecy analysis of ambient backscatter NOMA systems under I/Q imbalance," IEEE Transactions on Vehicular Technology, vol. 69, no. 10, pp. 12286-12290, 2020.

[5] X. Li, Q. Wang, Y. Liu, T. A. Tsiftsis, Z. Ding, and A. Nallanathan, "Uav-aided multi-way NOMA networks with residual hardware impairments," IEEE Wireless Communications Letters, vol. 9, no. 9, pp. 1538-1542, 2020.

[6] X. Li, Q. Wang, M. Liu et al., "Cooperative wireless-powered NOMA relaying for B5G IoT networks with hardware impairments and channel estimation errors," IEEE Internet of Things Journal, vol. 8, 2020.

[7] M. Zhan, J. Wu, H. Wen, and P. Zhang, "A novel error correction mechanism for energy-efficient cyber-physical systems in smart building," IEEE Access, vol. 6, pp. 3903739045, 2018.

[8] M. Zhan, Z. Pang, D. Dzung, and M. Xiao, "Channel coding for high performance wireless control in critical applications: survey and analysis," IEEE Access, vol. 6, pp. 29648-29664, 2018.

[9] IEEE, IEEE 802.15.4-2015 Standard, IEEE Standard for LowRate Wireless Networks, IEEE Press, New York, NY, USA, 2016.

[10] IEEE, Information Technology-Telecommunications and Information Exchange between Systems-Local and Metropolitan Area Networks-specific Requirements-Part 15-4: Wireless Medium Access Control (MAC) and Physical Layer (PHY) Specifications for Low-Rate Wireless Personal Area Networks (WPANs), IEEE Press, New York, NY, USA, 2018.

[11] T. Suzuki and T. Mizuno, "Multiple-symbol differential detection scheme for differential amplitude modulation," International Zurich Seminar on Digital Communications: Mobile Communications: Advanced Systems Components, Springer-Verlag, Berlin, Germany, 1994.

[12] C. Xu, S. X. Ng, and L. Hanzo, "Multiple-symbol differential sphere detection and decision-feedback differential detection conceived for differential QAM," IEEE Transactions on Vehicular Technology, vol. 65, no. 10, pp. 8345-8360, 2015.

[13] S. G. Wilson, J. Freebersyser, and C. Marshall, "Multi-symbol Detection of M-DPSK," 1989 IEEE Global Telecommunications Conference and Exhibition "Communications Technology for the 1990s and beyond", Dallas, TX, USA, 1989.

[14] V. Lottici and Z. Tian, "Multiple symbol differential detection for UWB communications," IEEE Transactions on Wireless Communications, vol. 7, no. 5, pp. 1656-1666, 2008.

[15] J. Jalden and B. Ottersten, "On the complexity of sphere decoding in digital communications," IEEE Transactions on Signal Processing, vol. 53, no. 4, pp. 1474-1484, 2005.

[16] A. Schenk and R. F. H. Fischer, "Compressed-sensing (decision-feedback) differential detection in impulse-radio ultrawideband systems," IEEE Transactions on Communications, vol. 59, no. 6, pp. 121-125, 2011.

[17] Y. Li, J. Wei, X. Wang, and Q. Yu, "Multiple symbol differential detection based on sphere decoding for unitary space-time modulation," Science in China Series F: Information Sciences, vol. 52, no. 1, pp. 126-137, 2009.

[18] IEEE, ISO/IEC/IEEE 802.15.4-2018 Standard, Information Technology-Telecommunications and Information Exchange between Systems-Local and Metropolitan Area Networksspecific Requirements-Part 15-4: Wireless Medium Access Control (MAC) and Physical Layer (PHY) Specifications for Low-Rate Wireless Personal Area Networks (WPANs), IEEE Press, New York, NY, USA, 2018.

[19] K.-H. Lin, W.-H. Chiu, and J.-D. Tseng, "Low-complexity architecture of carrier frequency offset estimation and compensation for body area network systems," Computers \& Mathematics with Applications, vol. 64, no. 5, pp. 1400-1408, 2012.

[20] H. J. Jeon, T. Demeechai, W. G. Lee et al., "IEEE 802.15.4 BPSK receiver architecture based on a new efficient detection scheme," IEEE Transactions on Signal Processing, vol. 58, no. 9, pp. 4711-4719, 2010. 
[21] G. Zhang, H. Wen, L. Wang, L. Song, J. Tang, and R. Liao, "Simple and robust near-optimal single differential detection scheme for IEEE 802.15.4 BPSK receivers," IET Communications, vol. 13, no. 2, pp. 186-197, 2019.

[22] J. L. Buetefuer and W. G. Cowley, "Frequency offset insensitive multiple symbol detection of MPSK," in Proceedings of the 2000 IEEE International Conference on Acoustics, Speech, and Signal Processing, Istanbul, Turkey, June 2000.

[23] S. Kay and P. Hall, "Fundamentals of statistical signal processing, volume ii:detection theory," Technometrics, vol. 37, no. 4, pp. 465-466, 1993.

[24] M. Luise and R. Reggiannini, "Carrier frequency recovery in all-digital modems for burst-mode transmissions," IEEE Transactions on Communications, vol. 43, no. 2/3/4, pp. 1169-1178, 1995.

[25] G. Zhang, D. Wang, L. Song et al., "Simple non-coherent detection scheme for IEEE 802.15.4 BPSK receivers," Electronics Letters, vol. 53, no. 9, pp. 628-629, 2017.

[26] G. Zhang, H. Wen, L. Wang et al., "Multiple symbol differential detection scheme for IEEE 802.15.4 BPSK receivers," IEICE Transactions on Fundamentals of Electronics, Communications and Computer Sciences, vol. E101.A, no. 11, pp. 1975-1979, 2018.

[27] G. Zhang, C. Shi, C. Han et al., "Implementation-friendly and energy-efficient symbol-by-symbol detection scheme for IEEE 802.15.4 O-QPSK receivers," IEEE Access, vol. 8, pp. 158402-158415, 2020.

[28] M. R. Bhatnagar, R. Vishwanath, M. K. Arti, and V. Bhatnagar, "A new iterative decoder of space-time data in time selective mimo channels," Wireless Personal Communications, vol. 42, no. 1, pp. 131-142, 2007.

[29] M. R. Bhatnagar, R. Vishwanath, and V. Bhatnagar, "Performance analysis of space-time block codes in flat fading mimo channels with offsets," EURASIP Journal on Wireless Communications and Networking, vol. 2007, no. 1, p. 7, 2007.

[30] M. R. Bhatnagar, R. Vishwanath, and M. K. Arti, "On blind estimation of frequency offsets in time varying MIMO channels," in Proceedings of the 2006 IFIP International Conference on Wireless and Optical Communications Networks, Banglore, India, April 2006.

[31] M. R. Bhatnagar and R. Vishwanath, "A new semiblind scheme based on second and fourth order statistics for decoding of space-time data in time selective MIMO channels," Wireless Personal Communications, vol. 47, no. 2, pp. 193-205, 2008.

[32] D. Park, C. S. Park, and K. Lee, "Simple design of detector in the presence of frequency offset for IEEE 802.15.4 LRWPANs," IEEE Transactions on Circuits and Systems II: Express Briefs, vol. 56, no. 4, pp. 330-334, 2009.

[33] J. H. Do, J. S. Han, H. J. Choi et al., "A Coherent DetectionBased Symbol Detector Algorithm for $2.45 \mathrm{GHz}$ LR-WPAN Receiver," in Proceedings of the Tencon 2005-2005 IEEE Region 10 Conference, Melbourne, Australia, November 2005.

[34] M. R. Bhatnagar and A. Hjorungnes, "SER Expressions for Double Differential Modulation," in Proceedings of the 2007 IEEE Information Theory Workshop on Information Theory for Wireless Networks, Bergen, Norway, January 2007.

[35] M. R. Bhatnagar, A. Hjorungnes, and L. Song, "Cooperative communications over flat fading channels with carrier offsets: a double-differential modulation approach," Eurasip Journal on Advances in Signal Processing, vol. 2008, no. 1, p. 11, 2008.

[36] M. Bhatnagar, A. Hjorungnes, and L. Song, "Double-differential orthogonal space-time block codes for arbitrarily correlated Rayleigh channels with carrier offsets," IEEE Transactions on Wireless Communications, vol. 9, no. 1, pp. 145-155, 2010.

[37] M. R. Bhatnagar and O. Tirkkonen, "Pl decoding in double differential modulation based decode-and-forward cooperative system," IEEE Communications Letters, vol. 17, no. 5, pp. 860-863, 2013. 